\title{
Clinical outcomes of hyperbaric oxygen therapy in treatment of postoperative chronic Pseudomonas aeruginosa wound infection following implant reconstruction of the breast
}

\author{
Tomasz Nowikiewicz ${ }^{1,2}$, Maria Szymankiewicz ${ }^{3}$, Barbara Zegarska ${ }^{4}$, Marta Biedka $^{5}$, Beata Nakonowska ${ }^{3}$, \\ Magdalena Nowikiewicz ${ }^{6}$, Wojciech Zegarski ${ }^{1}$
}

\begin{abstract}
${ }^{1}$ Chair and Department of Surgical Oncology, Collegium Medicum in Bydgoszcz, Nicolaus Copernicus University in Torun, Poland ${ }^{2}$ Department of Clinical Breast Cancer and Reconstructive Surgery, Prof. Franciszek Łukaszczyk Oncology Center, Bydgoszcz, Poland ${ }^{3}$ Department of Microbiology, Prof. Franciszek Łukaszczyk Oncology Center, Bydgoszcz, Poland

${ }^{4}$ Department of Cosmetology and Esthetic Dermatology, Collegium Medicum in Bydgoszcz, Nicolaus Copernicus University in Torun, Poland

${ }^{5}$ Chair and Department of Oncology and Brachytherapy, Collegium Medicum in Bydgoszcz, Nicolaus Copernicus University in Torun, Poland

${ }^{6}$ Student Scientific Society, Chair and Department of Surgical Oncology, Collegium Medicum in Bydgoszcz, Nicolaus Copernicus University in Torun, Poland
\end{abstract}

Infection is one of the most common causes of failure of generative treatment for breast cancer patients who have undergone mastectomy. Surgical site infection (SSI) in patients requiring the use of a synthetic breast implant can currently be observed in about $1-53 \%$ of cases [1, 2].

The most often isolated bacterial strains responsible for the occurrence of SSI are saprophytic Gram-positive bacteria (49-67\%) [1, 3-6]. Pathogens belonging to opportunistic in-hospital flora are Gram-negative rods or fungal wound infections, which may lead to complications [1, 4, 7]. SSI caused by these is characterized by a more severe course and a longer period of necessary treatment.

This paper describes the case of a patient who underwent a mastectomy with simultaneous breast reconstruction using the final prosthesis, which was complicated by the occurrence of SSI due to infection of the wound by a strain of Pseudomonas aeruginosa.

A 49-year-old patient was admitted to hospital in October 2017 for the surgical treatment of lesions in both breasts with atypical ductal hyperplasia (diagnosed via core needle biopsy) - a benign proliferative breast disease with a high frequency of coexisting cancer [8]. The woman had breast-conserving treatment (BCT) in June 2015 for left ductal carcinoma in situ (DCIS), with postoperative complementary 3D radiotherapy.
Histopathologic examination following quadrantectomy of both breasts revealed bilateral multifocal DCIS (with positive surgical margin). The patient was diagnosed with local recurrence in the left breast. The healing process of both wounds was free of complications.

The patient was referred to bilateral mastectomy with a sentinel node biopsy and simultaneous breast reconstruction (prosthesis Polytech, $445 \mathrm{ml}$ ).

On 6 December 2017, a left-sided skin and nipple-sparing mastectomy was performed with routine prophylaxis of antibiotic therapy (2.0 g cefazolin injection for 5 days).

Complications arose with the appearance of ischaemic symptoms on the third day after surgery, followed by marginal necrosis in the lateral lobe (Figure $1 \mathrm{~A}$ ). In addition to on-going antibiotic prophylaxis, the patient received pentoxifylline. On the fifth day local stability was achieved with partially demarcated necrotic tissues. After 2 weeks the patient was discharged to continue the healing process in an outpatient setting.

Subsequent check-ups revealed gradual progression of ischemic-necrotic changes (Figure $1 \mathrm{~B}$ ), the implant and necrotic tissues were removed, with a partial approximation of the wound edges (17 January 2018). Bacteriological studies confirmed the presence of Pseudomonas aeruginosa and Enterococcus faecalis and an abundant

Address for correspondence: Tomasz Nowikiewicz MD, Chair and Department of Surgical Oncology, Collegium Medicum, Nicolaus Copernicus University, 2 Romanowskiej St, 85-796 Bydgoszcz, Poland, phone: +48 $602534912,+48523743383$, e-mail: tomasz.nowikiewicz@gmail.com Received: 13.03.2019, accepted: 7.07.2019. 

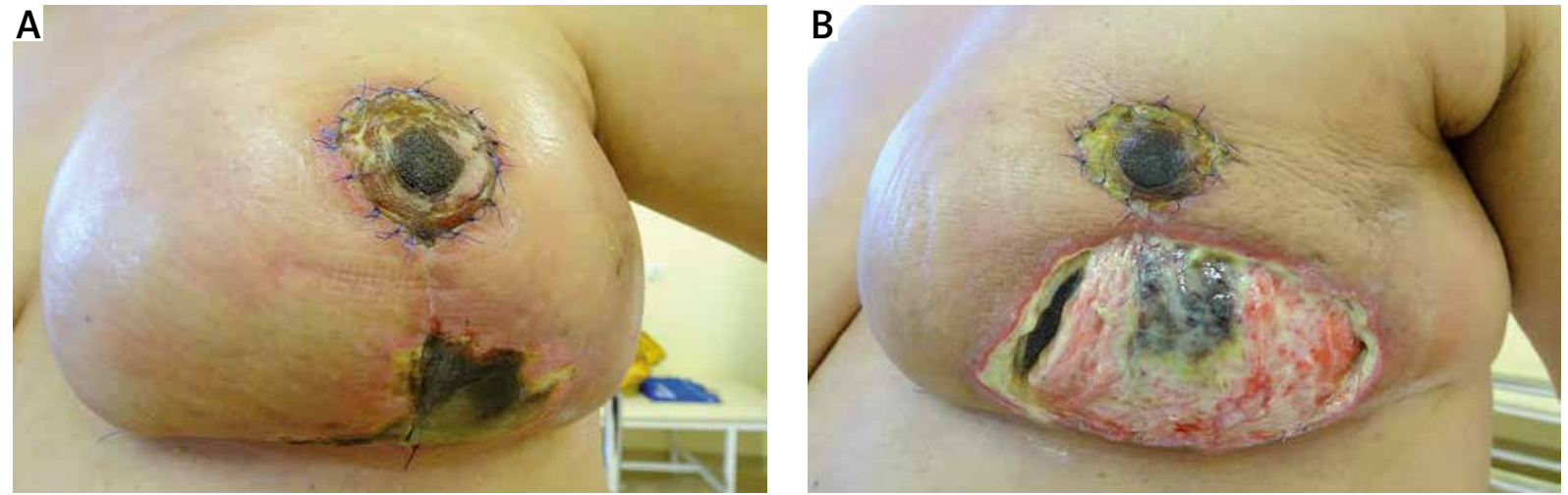

Figure 1. A - The marginal necrosis in the lateral lobe of the operative wound. B - The progression of ischemic-necrotic changes

increase in bacterial colonies. The patient received amikacin $1 \times 1.5 \mathrm{~g}+$ tazocin $3 \times 4.5 \mathrm{~g}$ i.v. (for 12 days), improving the local condition. In bacteriological examinations of wound secretions, negative cultures in aerobic conditions were obtained. The patient was discharged and continue the healing in an outpatient setting.

Due to the re-intensification of inflammation, a bacteriological study was taken. A large increase in the bacterial colonies of Pseudomonas aeruginosa was detected. Antibacterial treatment (ceftazidime $3 \times 2.0 \mathrm{~g}$, for 15 days) was introduced in the hospital setting. Aerobic bacteriological examinations yielded a negative result (24 March 2018).

The recurrence of Pseudomonas aeruginosa infection (30 March 2018) was observed. This ruled out the possibility of the scheduled surgery for the patient (reconstruction of the wound area).

Since targeted antimicrobial therapy proved ineffective twice, we decided it was necessary to implement treatment aimed at increasing tissue perfusion within the irradiated tissues of the wound region. The patient

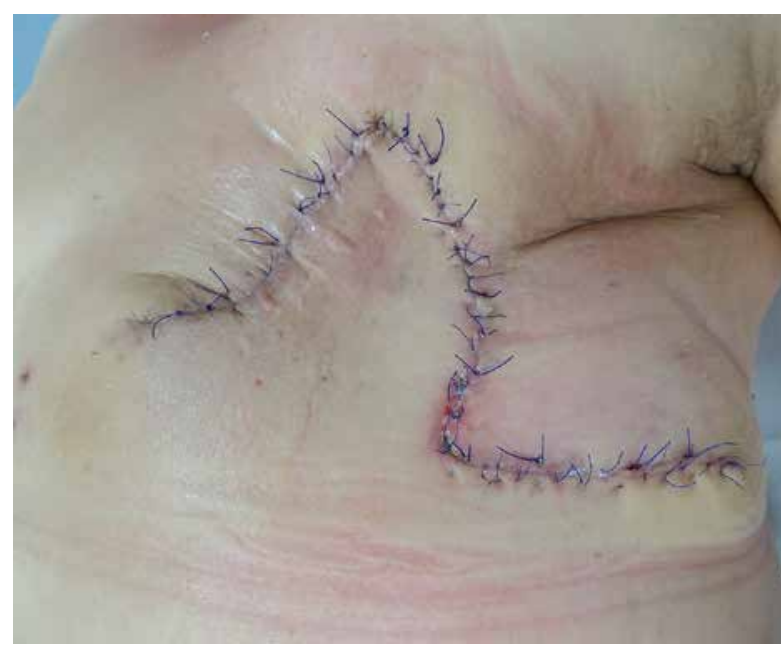

Figure 2. Postmastectomy wound - the final effect qualified for hyperbaric oxygen therapy. In the period between 19 April and 12 June 2018, a total of 30 treatments were performed (2.5 ATA/90 min), resulting in macroscopic improvement of the local condition. In a bacteriological study (9 June 2018), the abundant growth of Pseudomonas aeruginosa colonies continued.

Another hospitalization (18 June-17 July 2018) included targeted antibacterial treatment (ceftazidime $3 \times 2.0$ g i.v. + ciprofloxacin $3 \times 0.4$ g i.v., for 29 days) The bacteriological test yielded a negative culture result. On 6 July 2018 an operation was performed with extensive removal of necrotic tissues and scarred edges of the skin around the wound and closure of the wound (using rotating panels - Figure 2). In the postoperative period, no complications were observed.

The risk factors found in the described case (obesity, nicotinism, significant breast size, previous breast surgery, mastectomy with nipple conservation), significantly increase the probability of SSI in breast reconstruction using implants [1-3, 5, 6]. Factors also include microvascular alterations following radiotherapy of the breast $[9,10]$.

In order to reduce the incidence of SSI, pre-operative antibiotic prophylaxis is necessary $[4,7,10]$. According to Phillips et al., its introduction reduces the risk of infection (from $14.4 \%$ to $5.8 \%$ ) [11].

In the described case, such precautions were taken. Prolonged antibiotic prophylaxis (initiated before the patient's anaesthesia for surgery, continuing for 5 days) was used. However, this did not give the desirable result. As reported, clinical presentation was dominated by symptoms suggesting primarily insufficient blood supply to the produced dermal and subcutaneous flaps. This was due to the early onset of marginal necrosis, without the presence of other typical features of SSI.

The type of identified pathogenic flora is one of factors causing severe SSI [4, 12]. Another Pseudomonas aeruginosa infection issue concerns possible rapid antibiotic resistance to the type of treatment used to date [12]. As demonstrated in the bacteriological findings, in the analysed case, the encountered strain remained susceptible 
to the initially determined types of antibiotics at all times. This ultimately enabled it to be effectively eradicated.

In addition to removal of the implant, the finding of SSI makes it necessary to introduce targeted antibiotic therapy. According to current recommendations, this requires combined therapy of antibacterial drugs with a broad spectrum of activity [1, 2, 4]. In the group of patients analysed by Seng et al., the average period of antibiotic therapy was 44 days (range: 14-90 days) [4].

In the presented case, long-term antibiotic therapy was also used. However, in the first stage of treatment (for 14 days) this did not lead to eradication of the cause of SSI. Therefore, in accordance with results of studies on the clinical effects of hyperbaric oxygen therapy in patients after radiotherapy on their breasts during BCT $[13,14]$, the patient was additionally given this form of treatment. The resulting improvement in the state of local blood supply to tissues (assessed only macroscopically), together with repeated antibacterial treatment (for a period of 29 days), finally allowed the wounds to heal.

\section{Conflict of interest}

The authors declare no conflict of interest.

\section{References}

1. Pittet B, Montandon D, Pittet D. Infection in breast implants. Lancet Infect Dis 2005; 5: 94-106.

2. Rubino C, Brongo S, Pagliara D, et al. Infections in breast implants: a review with a focus on developing countries. J Infect Dev Ctries 2014; 8: 1089-95.

3. Kato H, Nakagami G, Iwahira Y, et al. Risk factors and risk scoring tool for infection during tissue expansion in tissue expander and implant breast reconstruction. Breast J 2013; 19: 618-26.

4. Seng P, Bayle S, Alliez A, et al. The microbial epidemiology of breast implant infections in a regional referral centre for plastic and reconstructive surgery in the south of France. Int J Infect Dis 2015; 35: 62-6.

5. Francis SH, Ruberg RL, Stevenson KB, et al. Independent risk factors for infection in tissue expander breast reconstruction. Plast Reconstr Surg 2009; 124: 1790-6.

6. Nahabedian MY, Tsangaris T, Momen B, et al. Infectious complications following breast reconstruction with expanders and implants. Plast Reconstr Surg 2003; 112: 467-76.

7. Weichman KE, Levine SM, Wilson SC, et al. Antibiotic selection for the treatment of infectious complications of implant-based breast reconstruction. Ann Plast Surg 2013; 71: 140-3.

8. Hodorowicz-Zaniewska D, Brzuszkiewicz K, Szpor J, et al. Clinical predictors of malignancy in patients diagnosed with atypical ductal hyperplasia on vacuum-assisted core needle biopsy. Videosurgery Miniinv 2018; 13: 184-91.

9. Agrawal A. Oncoplastic breast surgery and radiotherapyadverse aesthetic outcomes, proposed classification of aesthetic components, and causality attribution. Breast J 2019; 25: 207-18.

10. Jagsi R, Jiang J, Momoh A, et al. Complications after mastectomy and immediate breast reconstruction for breast cancer: a claims-based analysis. Ann Surg 2016; 263: 219-27.
11. Phillips BT, Bishawi M, Dagum AB, et al. A systematic review of antibiotic use and infection in breast reconstruction: what is the evidence? Plast Reconstr Surg 2013; 131: 1-13.

12. Pobiega M, Chmielarczyk A, Koziol J, et al. Virulence factors genes and drug resistance in Pseudomonas aeruginosa strains derived from different forms of community and healthcare associated infections. Postepy Hig Med Dosw 2018; 72: 751-9.

13. Teguh DN, Bol Raap R, Struikmans H, et al. Hyperbaric oxygen therapy for late radiation-induced tissue toxicity: prospectively patient-reported outcome measures in breast cancer patients. Radiat Oncol 2016; 11: 130.

14. Tahir AR, Westhuyzen J, Dass J, et al. Hyperbaric oxygen therapy for chronic radiation-induced tissue injuries: Australasia's largest study. Asia Pac J Clin Oncol 2015; 11: 68-77. 